\title{
Aqua-Fi: Delivering Internet Underwater using Wireless Optical Networks
}

\author{
Basem Shihada, Osama Amin, Christopher Bainbridge, Seifallah Jardak, Omar Alkhazragi, \\ Tien Khee Ng, Boon Ooi, Michael Berumen, and Mohamed-Slim Alouini
}

\begin{abstract}
In this article, we demonstrate bringing the Internet to underwater environments by deploying a low power and compact underwater optical wireless system, called Aqua-Fi, to support today's Internet applications. Aqua-Fi uses a lightemitting diode (LED) or Laser to support bidirectional widerange communication services with different requirements, low cost, and simple implementation. LEDs introduce robust short distances solutions with low power requirements. However, Laser extends the communication distance and improves the transmission rate at the cost of higher power requirements. Throughout this work, we discuss the proposed Aqua-Fi system architecture, limitations, and solutions to improve data rates and deliver reliable communication links.
\end{abstract}

\section{INTRODUCTION}

By 2030, there will be more than 40 billion devices simultaneously connected to the Internet, including computers, smart devices, machines, and eventually autonomous vehicles. Over the years, the Internet has proven to be able to absorb the increasing demands and continue to function reliably. As of today, the Internet is capable of connecting almost everything, including transmitting media data beyond Earth to interstellar space, as we witnessed from both Voyager 1 and 2 space probes. On the other hand, there has been a noticeable limitation of Internet coverage when it comes to the underwater environment. The reasons vary from the harsh underwater environments to the physical-medium limitations.

Underwater wireless communication can be realized by three types of mediums: acoustic, radio-frequency (RF), and optical. Acoustic transmission can exchange data for long-ranges but at low data rates. On the other hand, RF transmissions can achieve high throughput but at short distances due to high absorption in water. Optical wireless transmissions introduce promising solutions by allowing high throughput but for medium ranges due to several underwater-environment challenges [1]. Firstly, the water absorbs and scatter small fractions of the optical wave, which depends on the water type. Secondly, the refraction index can vary along the propagation path due to fluctuations in

The authors are with the Computer, Electrical and Mathematical Sciences \& Engineering (CEMSE) Division, King Abdullah University of Science and Technology (KAUST), Thuwal 23955, Makkah Province, Saudi Arabia.

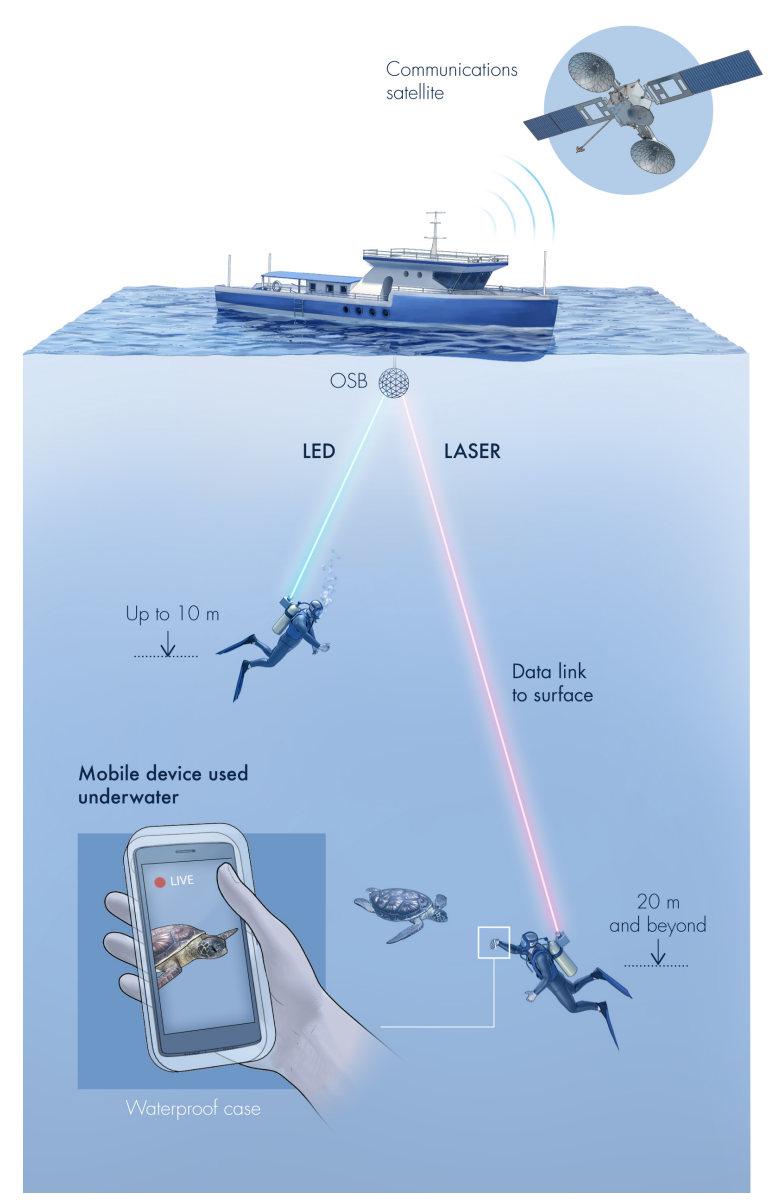

Fig. 1: High-level view of the Aqua-Fi architecture

the density, salinity, and temperature variation across the underwater environment. Thirdly, optical beams are very narrow, and thus the line-of-sight must be maintained for a reliable link performance. Fourthly, the noise from other light sources, including the Sun, can have a degradation performance effect, especially in the offshore areas. Fifthly, underwater objects can play the role of scatters and reflectors, which results in multipath interference on the received optical signal at the detector side. For example, highly turbid underwater environments can cause time dispersion of optical signals, thus decreases the symbol data rate due to inter-symbol interference. Therefore, we need to adopt several communication techniques to mitigate multipath interference, such as spatial diversity. An- 
other serious problem is the link outage due to marine animals, where optical signals attract marine animals and can cause momentary loss of signal at the receiver. Appropriate error correction, signal processing, and redundancy techniques are required to ensure delivering the data correctly.

In this article, we propose, Aqua-Fi system, as a first significant step towards realizing the vision of bringing the Internet in underwater environments, as shown in Fig. 1. Aqua-Fi incorporates an Internet bridge, a transmission optical modulator, transmitter equipped with projection optics, beam steering system, detector, and then a subsequent signal processing unit for demodulation. Aqua-Fi system has several practical advantages, which are summarized as follows:

- Cost-Effective: Aqua-Fi can use low power offthe-shelf components such as LEDs and Lasers to transmit Internet data with rates in Mbps range over a few meters in underwater environments.

- Flexible: Aqua-Fi relies on multi-hop communication using different technologies and communication nodes capabilities, as shown in Fig. 1.

- Practical: Aqua-Fi does not require any additional underwater infrastructure, where it can operate using self-contained batteries with low powered light communication devices. Moreover, it leverages standard packet-based communication protocols; thus, it could interoperate with other systems.

We propose two Aqua-Fi systems based on either LEDs or Lasers light sources, which are connected to Raspberry Pis at each connecting end. The LEDs based system utilizes cost-effective materials with small size and operates at low power, but with limited range. However, the Aquai-Fi laser-based system can achieve a higher range and throughput at the cost of weight, size, and power requirements compared with the LEDs one.

In the rest of this article, we first give an overview of the existing experimental and commercial optical underwater systems in addition to the terrestrial opticalbased systems. Then, we discuss the main features of the underwater Internet network. Later, we propose and describe Aqua-Fi system component, followed by a discussion of the system's performance and limitations. Finally, we summarize and provide a comprehensive discussion about future work.

\section{OpticAl COMMUNiCATION Systems}

Researchers in both academy and industry are spending extensive efforts to develop experimental and commercial optical systems in both terrestrial and underwater environments [2]. In this section, we highlight the last advances in commercial and experimental underwater communication systems. Then, we discuss some efforts on terrestrial optical-based in order to borrow some approaches to the underwater environment after considering necessary modifications.

\section{Experimental Systems}

Several lab experiments are conducted for underwater optical communication (UWOC) systems to test point-to-point communication for different ranges [3][7] As such, the wavelengths from $450 \mathrm{~nm}$ to $550 \mathrm{~nm}$ that represent the visible spectrum between the blue and green regions are chosen for their lowest attenuation performance [2], [8]. Recently, real-time video transmission using Quadrature-Amplitude Modulation (QAM) up to 8-QAM in different water types was demonstrated in [6]. Moreover, the aforementioned one-way communication experiment was extended to a bi-directional point-to-point communication scenario using laser technology in [9]. Furthermore, higher resolutions of underwater video that requires high data rate are achieved using multi-carrier OrthogonalFrequency Division-Multiplexing, where high-orders of QAM signal is used at each sub-carrier to utilize the spectrum efficiently [3], [7]. Table I illustrates some of the systems that have been developed and experimented in the lab using different power and technologies and provide varied services quality in terms of data rate and communication range. According to the lab experiments in [3], [10]-[14], one can find that simple On-Off Keying (OOK) modulation can achieve an acceptable performance. Also, Laser-based systems can provide a much longer range than LED-based ones at the cost of power and system complexity. Furthermore, high data rates (gigabit) have not been observed for vast distances. Finally, orthogonal frequency-division multiplexing employing link diversity can achieve further distances with high data rates. In all of these cases, beam alignment is critical and complicated, where it requires sophisticated hardware support.

\section{Commercial Systems}

On the practical side, several companies introduced underwater communication solutions between two nods/users, such as Bluecomm, Anglerfish, and Divecomm. Bluecomm utilizes the LED lights in underwater equipment, such as remotely operated vehicles, to establish communication links. It utilizes a high power LED that operates in shallow or deep water, depending on the application. The reported data rates vary with the communication distance, where a rate of up to 500 Mbps is achieved for very short distances; however, 5 - 10 Mbps can be achieved for longer distances. Anglerfish provides full-duplex voice communication between two divers using also LED technology. It is designed for military applications, with the claim that it 
TABLE I: Results from Underwater Lab Testbeds

\begin{tabular}{|l|c|c|c|c|c|c|}
\hline Data Rate & Range & Spectrum & Modulation & Receiver & Transmitter & Power \\
\hline$[3] 4.8 \mathrm{Gbps}$ & $5.4 \mathrm{~m}$ & $450 \mathrm{~nm}$ & 16-QAM OFDM & APD & Laser & $15 \mathrm{~mW}$ \\
{$[10] 1 \mathrm{Gbps}$} & $2 \mathrm{~m}$ & $532 \mathrm{~nm}$ & OOK & APD & Laser & $10 \mathrm{~mW}$ \\
{$[11] 1 \mathrm{Mbps}$} & $3 \mathrm{~m}$ & $532 \mathrm{~nm}$ & BPSK & PMT & Laser & $3 \mathrm{~W}$ \\
{$[12] 2.3 \mathrm{Gbps}$} & $7 \mathrm{~m}$ & $520 \mathrm{~nm}$ & OOK & APD & Laser & $12 \mathrm{~mW}$ \\
{$[13] 2.28 \mathrm{Mbps}$} & $50 \mathrm{~m}$ & $470 \mathrm{~nm}$ & DPIM & APD & LED & $10 \mathrm{~W}$ \\
{$[14] 161 \mathrm{Mbps}$} & $2 \mathrm{~m}$ & $450 \mathrm{~nm}$ & 16-QAM OFDM & APD & LED & N/A \\
\hline
\end{tabular}

is difficult to intercept because of the directionality and unknown modulation frequencies. Lastly, Divecomm tested a system in early 2017 that achieved $100 \mathrm{Mbps}$ over a range of 30 meters. It uses mirrors to focus the received light into the photodetector. However, their last update recorded a failure to operate in seawater at 100 meters. It is worthy to note that the existing experimental and commercial UWOC system focuses only on point-to-point connections and does not show any network implementation, including the Internet. In the following section, we explore the advances in terrestrial optical-based communication systems to learn some lessons that may help in developing an Internet connection using underwater wireless optical communication.

\section{Terrestrial optical-based systems}

$\mathrm{Li}-\mathrm{Fi}$ is a terrestrial optical system that uses visible light technology based on standard 802.11 protocols and transfers data via the visible spectrum. Currently, PureLifi is the only commercial system in the market, where it offers an integrated device (PL0300) implementing an 802.11 compatible stack with a USB interface supporting up to $86.4 \mathrm{Mbps}$ data rate. Infrared Data Association provides a specification for gigabit transmissions using infrared light, where the IrLAN protocol allows connecting to local area networks. However, it has dropped out of use, and there are not any available modules on the market anymore. Small form-factor pluggable (SFP) is a widespread optical fiber interface that is mainly used in network switches and can achieve beyond 10 Gbps transmission rate. Koruza is an open-source implementation of a Free Space Optical (FSO) system that uses off-the-shelf SFP modules operating at the infra-red light frequencies and achieving up to $1 \mathrm{Gbps}$ data rate. It is worth noting that currently, there are not available SFP transceivers operating at the $450-520 \mathrm{~nm}$ visible-light range.

\section{UNDERWATER NETWORK FEATURES}

In this section, we discuss the key aspects of the proposed underwater Internet solution that aims at facilitating network deployment in the underwater environment and using today's Internet applications for text and multimedia.

\section{Flexibility}

A vital feature of the proposed underwater Internet network solution is to be flexible where handheld devices can operate freely underwater with maximum bidirectional bandwidth. To provide the basis for this point, we consider an abstract model of a diver using a handheld device that can send text and multimedia information. We use different wireless technologies to have additional flexibility degrees while forming a multi-hop communication network. Several sorts of data at the diver side can be transferred first to a gateway attached to his back and equipped with different network interfaces such as $\mathrm{WiFi}$ and optical ones.

\section{Wireless Links}

To realize the previously mentioned flexible network, we need high bandwidth multi-hop wireless links between the divers and buoys or any other underwater communication nodes, as shown in Fig. 1. The straightforward solution would be to use RF wireless links that is suitable for short distances [2], [8]. Thus, we can use it for the link between the diver's handheld device and the main gateway, as depicted in Fig. 2. Then, the gateway relays the received signal to the nearest receiver using LED or Laser transceivers, which can accommodate high throughput for long distances. Furthermore, the proposed system should communicate vertically between the diver and surface buoy or horizontally between divers using the same optical technology. Finally, the last hop is then from the ship's receiver to the Internet via satellite or any alternative terrestrial-based wireless connection.

\section{Access to Internet Applications}

Realizing today's various Internet applications and unleashing their potentials necessitate a paradigm shift in the way current underwater networks operate. Previous underwater video streams systems [9] is incapable of being used as general-purpose wireless connections. Our particular focus is on utilizing standardized protocols to allow accessing all Internet applications and interoperating with different wireless systems in the network. Since most of these applications would require end-to-end performance guarantees, we need 


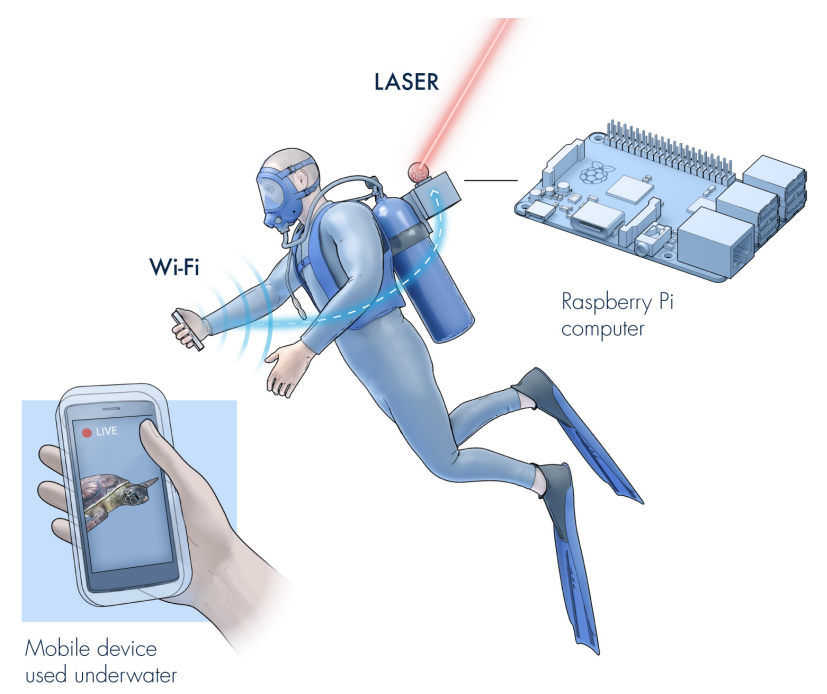

Fig. 2: Aqua-Fi System Overview

to alleviate different challenges for the underwater wireless network. Underwater physical medium limits the massive bandwidth, power, and computation requirements. Multi-hop relaying across multiple buoys is a powerful solution to distribute the burden among several network nodes. Furthermore, joint context awareness at the underwater wireless and the core network is fundamental to provide adequate prioritization, reliability, and security for the coexisting applications.

\section{AquA-Fi System OVERVIEW}

The ultimate goal of our system is to provide a high-speed point-to-point UWOC link utilizing both LEDs and lasers depending on operation requirements, as shown in Fig. 1. First, we construct the Aqua-Fi system using LEDs, which is suitable for short distances and low power requirements. Then, we extended the transmission distance and throughput by employing laser sources. Both setups are implemented in a static environment with negligible turbulence.

\section{Aqua-Fi Raspberry Pi-Based LED System}

We build the system firstly utilizing green LEDs $(520 \mathrm{~nm})$ as the transmitters, with photodiodes as the receivers (DET10A2 from Thorlabs). We connect each transmitter to the Universal asynchronous receiver/transmitter (UART) transmit line of a Raspberry Pi 3B via a Bias Tee. As for the receiver side, we connect it also to the receiving UART line of the Raspberry Pi via an amplifier and comparator. Then, we construct the other link, in the same way, to develop a full-duplex symmetric communication system, as shown in Fig. 3.

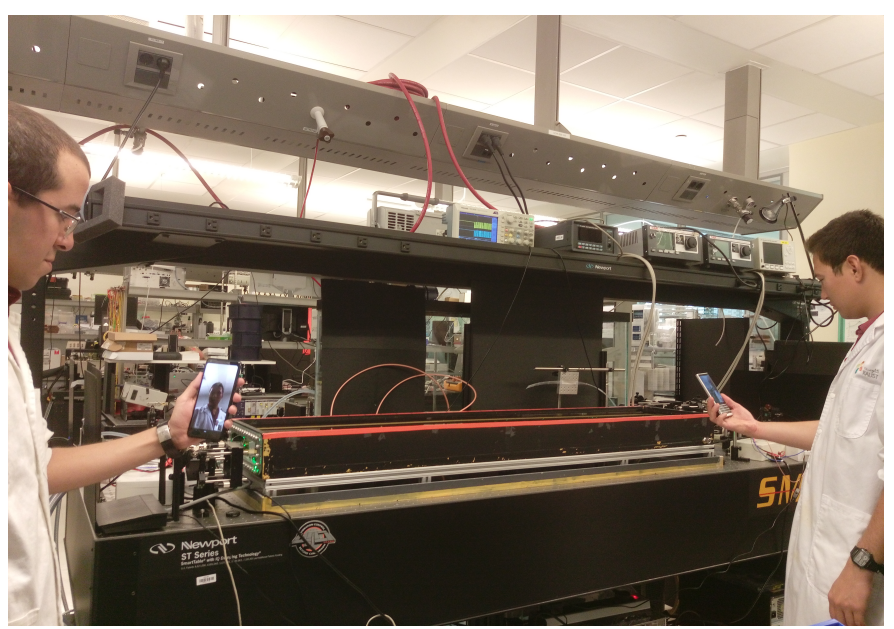

Fig. 3: Aqua-Fi setup in a lab environment showing a video call being made and data capture on the oscilloscope

Software: We use the point-to-point protocol (PPP) to modulate the optical signal using OOK through the UART link. As for the transmitting Raspberry $\mathrm{Pi}$, we configure it to forward the Ethernet interface to the PPP interface, allowing a connection to the Internet. The remote Raspberry Pi forwards the packets again from the PPP interface to the Ethernet interface that is connected to an external access point. As for the mobile phone or the tablet connects to the access point via $\mathrm{WiFi}$, allowing phone connection to the Internet. The Ethernet and PPP interfaces are bridged using IPv4 forwarding and iptables; dhcpd is provided at the access point end to assign the hand-held device an IP address automatically. The system is used to start the processes on boot, as per the standard way of doing system initialization.

\section{Asymmetric Power Availability}

The Aqua-Fi design considers different power requirements and availability. For underwater nodes, where low power requirement is necessary, we use a transmitter that operates at $5 \mathrm{~V}$. As for the detection; we utilize the DET10A2 photodiode that operates with low power and gives an output signal from 0 to $80 \mathrm{mV}$. On the other hand, the transmitter at the surface nodes operates with $12 \mathrm{~V}$ battery to send a strong signal. The detector at the surface node utilizes the Thorlabs PDA10A2 receiver, which is a photodiode with an integrated trans-impedance amplifier, with higher power requirements; thus, it allows an output signal up to $1 \mathrm{~V}$.

\section{Aqua-Fi Laser System}

We modify the Aqua-Fi LED-based system with $0.3 \mathrm{~W} 520 \mathrm{~nm}$ SN-LDM-T-P TO-Can lasers from SaNoor Technologies. As can be seen from previous 


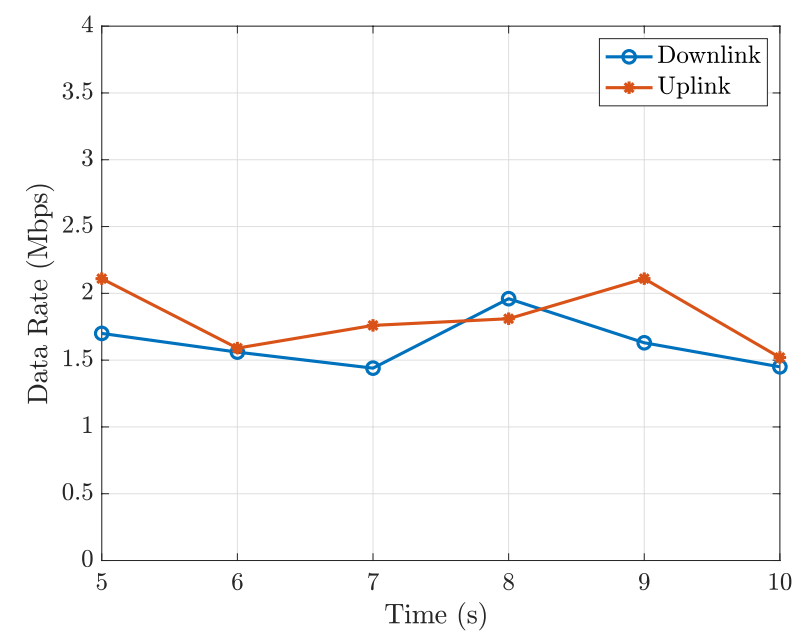

Fig. 4: Sum of four parallel data streams through uplink and downlink versus transmission time.

work in Table I, LED systems typically have a lower data rate due to their lower switching speeds, thus switching to lasers allow us to develop a faster system. The range of lasers is also considerably higher, but this is due to their higher power requirements compared to LEDs. They are also considerably more expensive and have much higher pointing requirements.

Software Changes: We were able to increase the UART link rate to $1 \mathrm{Mbps}$ using OOK transmission. The rest of the software was kept the same.

\section{System Performance}

We measure Aqua-Fi system performance by throughput, latency, and packet loss, assuming a static underwater channel and fully aligned optical beam with both the transmitter and receiver. First, we use four parallel streams, which denotes four different traffic carried via the Internet optical link. Then, we measure the throughput using iperf3, where the sum of the four streams can acheive a maximum of $2.11 \mathrm{Mbps}$, as shown in Fig. 4. Moreover, we observe approximate symmetry between both links, which verifies the link stability. As for the latency, we measured it using ping and observed the round-trip time for different packets, as shown in Fig. 5. At no load, the measured latency has an average of $1.03 \mathrm{~ms}$, while under stress test, it can increase up to $85.5 \mathrm{~ms}$. The delay increase is due to physical link performance because the CPU load was low as measured with top. As for the packet loss, it was $0 \%$ when we measured it with flood ping, it had no packet loss when we used iperf 3 and adopted the TCP test. When we increased the rate to $17 \mathrm{Mbps}$, UDP test reported packets loss.

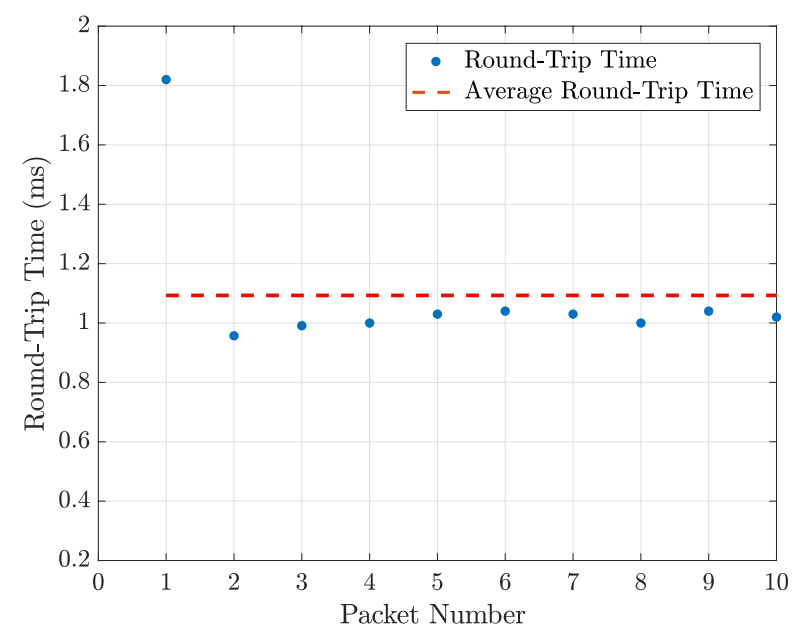

Fig. 5: Packet latency of Internet optical link.

\section{LIMITATIONS AND FUTURE WORK}

In this section, we shed light on factors that limit Aqua-Fi performance, followed by several future directions that we should consider to improve the system performance.

\section{Limitations}

The Aqua-Fi system implementation suffers from some challenges that limit the overall performance. The conducted experiment in the lab or pool environments owns static communication channels, which requires manual adjustment of the receiver amplifier. However, when the system is implemented in a real sea/ocean environment, any motion could result in nonnegligible turbulence that affects the communication channel quality and link alignment.

Although using Raspberry Pi allows employing several processing techniques for the signal and flexible control with other peripheral devices, the UART limits the output data rate performance in the megabit range. Raspberry $\mathrm{Pi}$ has a serial peripheral interface, interintegrated circuit, and UART connections, which are not suitable for a system targeting gigabit performance. Thus, it is necessary to find/develop alternatives with higher speed interfaces, that can process the data quickly enough as required. The system also needs to maintain a low power in order to function underwater with a limited room to mount batteries.

\section{Future Work}

Improving Data Rate: To improve the data rate significantly, we suggest replacing the Raspberry Pis with standard SFP transceivers driving the lasers/receivers, which is common in FSO systems. SFP is capable of transferring gigabit data rate and integrating with 
automatic gain controller (AGC) at the receiver, and with the automatic power control at the transmitter. Also, we can use a proper encoding scheme, such as $8 \mathrm{~b} / 10 \mathrm{~b}$, and utilize the benefits of Ethernet standards such as error-correcting codes, which do not exist in PPP. All of these are standard off-the-shelf components, keeping costs low.

In the SFP transceiver, we will replace the infrared lasers with $520 \mathrm{~nm}$ lasers, i.e., the same one used from SaNoor Technologies. One way to monitor the transmit power is by using an external photodiode. As for boosting the voltage and current to accommodate a higher power laser, we can use high-speed op-amps in a differential amplifier configuration connected to a constant current driver. As for the receiver, we will use visible light photodiodes with integrated transimpedance amplifiers (capable of $1.25 \mathrm{Gbps}$ ), which also integrate AGC functionality (part FCI-H125G-010 from OSI Optoelectronics). Moreover, we will need to use SFP to Ethernet converters (MC220L Gigabit Ethernet Media Converters from TP-Link) to bridge the optical network to Ethernet, which thus allows connections to an access point and the Internet.

Control System: Developing a robust control system to track the optical beam and align it with the receiver can affect the Aqua-Fi system performance significantly. Beam alignment can be done using either out-of-band or in-band techniques. The out-of-band method uses a different band for the control mechanism to localize and track each station. The acoustic signal can be used to track the source, where its low data rate is sufficient to do so. Another approach is by using the cameras to track the laser beam and adjust the alignment visually. As for the in-band system, the laser signal is used to ensure the best alignment angle by measuring the signal-to-noise ratio or received signal strength indicator values. Thus, when the inband system is required, it must be factored into the protocols used as time is needed to utilize the lasers for measurements.

Underwater Optical Receivers: Designing optical receivers with high response levels at visible light ranges is essential to obtain reliable links. Moreover, extending the single photodiode to a planar or spherical array would increase the received signal strength and reduce the pointing requirements. Furthermore, utilizing solar arrays enables harvesting energy besides transferring data by the optical beam and thus reduces the energy requirement. Solar cells are expected to participate effectively in the underwater optical communication, especially after releasing some restrictions on the bandwidth [15].

Miniaturization: The system as a whole requires miniaturizing to allow mounting it to small objects (such as human divers and mid-water sensors). Hence, it will also help to reduce the power consumption of the whole system.

\section{CONCLUSIONS}

In this article, we demonstrated our vision of Aqua$\mathrm{Fi}$ as a novel underwater communication system that facilitates generic Internet-connected applications. Aqua$\mathrm{Fi}$ is a flexible, low cost, and low power underwater network solution that utilizes Raspberry Pis with RF and optical transceivers. We built and tested the Aqual-Fi system to can connect an underwater platform wirelessly to the Internet and transmit real-time voice and video using both LEDs and $520 \mathrm{~nm}$ Laser in a static water environment. Our future efforts will focus on providing higher data rates and immunity against mobility by using a high-speed transceiver, highly reactive control system, and improved detection system to fulfill our vision of bringing the Internet underwater.

\section{ACKNOWLEDGMENT}

This work was funded by the Red Sea Research Center and Figures 1 and 2 were produced by Xavier Pita, scientific illustrator at King Abdullah University of Science and Technology (KAUST).

\section{REFERENCES}

[1] C. Gussen, P. Diniz, M. Campos, W. Martins, F. Costa, and J. Gois, "A survey of underwater wireless communication technologies," $J$. Commun. Inform. Syst., vol. 31, no. 1, pp. 242-255, 2016.

[2] N. Saeed, A. Celik, T. Y. Al-Naffouri, and M.-S. Alouini, "Underwater optical wireless communications, networking, and localization: A survey," Ad Hoc Netw., pp. 1-35, 2019.

[3] H. M. Oubei, J. R. Duran, B. Janjua, H.-Y. Wang, C.-T. Tsai, Y.-C. Chi, T. K. Ng, H.-C. Kuo, J.-H. He, M.-S. Alouini, G.R. Lin, and B. S. Ooi, "4.8 Gbit/s 16-QAM-OFDM transmission based on compact 450-nm laser for underwater wireless optical communication," Opt. Express, vol. 23, pp. 23 302-23 309, Sept. 2015.

[4] E. Zedini, H. M. Oubei, A. Kammoun, M. Hamdi, B. S. Ooi, and M.-S. Alouini, "Unified statistical channel model for turbulenceinduced fading in underwater wireless optical communication systems," IEEE Trans. Commun., vol. 67, no. 4, pp. 2893-2907, April 2019.

[5] M. Doniec, Anqi Xu, and D. Rus, "Robust real-time underwater digital video streaming using optical communication," in Proc.IEEE Int. Conf. Robotics and Automation, 2013, pp. 5117-5124.

[6] A. Al-Halafi, H. M. Oubei, B. S. Ooi, and B. Shihada, "Realtime video transmission over different underwater wireless optical channels using a directly modulated $520 \mathrm{~nm}$ laser diode," IEEE/OSA J. Opt. Commun. Netw., vol. 9, no. 10, pp. 826-832, 2017.

[7] Y. Chen, M. Kong, T. Ali, J. Wang, R. Sarwar, J. Han, C. Guo, B. Sun, N. Deng, and J. Xu, " $26 \mathrm{~m} / 5.5 \mathrm{Gbps}$ air-water optical wireless communication based on an OFDM-modulated 520-nm laser diode," Opt. Express, vol. 25, no. 13, pp. 14760-14765, June 2017.

[8] Z. Zeng, S. Fu, H. Zhang, Y. Dong, and J. Cheng, "A survey of underwater optical wireless communications," IEEE Commun. Surveys Tuts., vol. 19, no. 1, pp. 204-238, 2017.

[9] A. Al-Halafi and B. Shihada, "UHD video transmission over bidirectional underwater wireless optical communication," IEEE Photon. J., vol. 10, no. 2, pp. 1-14, April 2018.

[10] F. Hanson and S. Radic, "High bandwidth underwater optical communication," Appl. Opt., vol. 47, no. 2, pp. 277-283, Jan. 2008. 
[11] B. Cochenour, L. Mullen, A. Laux, and T. Curran, "Effects of multiple scattering on the implementation of an underwater wireless optical communications link," in Proc. OCEANS 2006, Sept. 2006, pp. 1-6.

[12] H. M. Oubei, C. Li, K.-H. Park, T. K. Ng, M.-S. Alouini, and B. S. Ooi, " $2.3 \mathrm{Gbit} / \mathrm{s}$ underwater wireless optical communications using directly modulated $520 \mathrm{~nm}$ laser diode," Opt. Express, vol. 23, no. 16, pp. 20743-20748, Aug 2015.

[13] M. Doniec and D. Rus, "BiDirectional optical communication with AquaOptical II," in Proc. IEEE Int. Conf. Commun. Syst., Nov. 2010, pp. 390-394.

[14] J. Xu, M. Kong, A. Lin, Y. Song, X. Yu, F. Qu, J. Han, and N. Deng, "OFDM-based broadband underwater wireless optical communication system using a compact blue LED," Opt. Commun., vol. 369, pp. $100-105,2016$.

[15] W.-H. Shin, S.-H. Yang, D.-H. Kwon, and S.-K. Han, "Selfreverse-biased solar panel optical receiver for simultaneous visible light communication and energy harvesting," Opt. express, vol. 24, no. 22, pp. A1300-A1305, 2016.

Basem Shihada [SM'12] (basem.shihada@kaust.edu.sa) received the Ph.D. degree in computer science from the University of Waterloo, Waterloo, ON, Canada. He is an Associate and a Founding Professor with the Computer, Electrical and Mathematical Sciences Engineering Division, King Abdullah University of Science and Technology, Thuwal, Saudi Arabia. In 2009, he was appointed as a Visiting Faculty Member with the Department of Computer Science, Stanford University, Stanford, CA, USA. His current research interests include energy and resource allocation in wired and wireless networks, software-defined networking, Internet of Things, data networks, smart systems, network security, and cloud/fog computing.

Osama Amin [SM'15] (osama.amin@kaust.edu.sa) received his B.Sc. degree in electrical and electronic engineering from Aswan University, Egypt, in 2000, his M.Sc. degree in electrical and electronic engineering from Assiut University, Egypt, in 2004, and his Ph.D. degree in electrical and computer engineering, University of Waterloo, Canada, in 2010. In June 2012, he joined Assiut University as an assistant professor in the Electrical and Electronics Engineering Department. Currently, he is a research scientist in the CEMSE Division at KAUST, Thuwal, Makkah Province, Saudi Arabia. His general research interests lie in communication systems and signal processing for communications with special emphasis on wireless applications.

Christopher Bainbridge (cebainbridge@ hotmail.com) graduated from the University of Bristol, UK in 2014 with an MEng Computer Science and Electronics with Study Abroad, 1st Class Honours. He was employed as an Embedded Software Engineer with Blu Wireless, based in Bristol, before joining KAUST to work on an Underwater Optical Wireless Communication system, where he helped to progress the speed and capabilities. He currently works in the MAC / Radio team as a Senior Embedded Software Engineer at Blu Wireless.

Seifallah Jardak (seifallah.jardak@kaust.edu.sa) received the Diplome d'Ingénieur degree from the École Polytechnique de Tunisie, La Marsa, Tunisia, in 2012, the Master and Ph.D of Science degree in electrical engineering from King Abdullah University of Science and Technology (KAUST), Thuwal, Saudi Arabia, in 2014 and 2018, respectively. His research interests include MIMO and passive radar, with focusing on the design and implementation of a portable low-cost FMCW radar.
Omar Alkhazragi [S'19] (omar.alkhazragi@kaust.edu.sa) received the Bachelor of Science degree in electrical engineering from the King Fahd University of Petroleum and Minerals, Dhahran, Saudi Arabia, in 2018. He is currently working toward the M.S. and Ph.D. de- grees in electrophysics with the Photonics Laboratory, King Abdullah University of Science and Technology, Thuwal, Saudi Arabia. His research interests include experimental and theoretical studies of optical wireless communication systems.

Tien Khee Ng [SM'17] (tienkhee.ng@kaust.edu.sa) received the M.Eng. and Ph.D. degrees from Nanyang Technological University, Singapore, in 2001 and 2005. He is a Senior Research Scientist with the King Abdullah University of Science and Technology, Thuwal, Saudi Arabia and a Co-Principal-Investigator responsible for innovation in MBE-grown nanostructures and devices with the KACST's Technology Innovation Center, KAUST, Thuwal, Saudi Arabia. His research focuses on the fundamental and applied research of wide-bandgap group-III nitride, novel hybrid materials and multi-functional devices for efficient lightemitters, optical wireless communication, and energy harvesting. He is also a Senior Member of OSA, Member of SPIE, and Member of IOP.

Boon S. Ooi (boon.ooi@kaust.edu.sa) received the B.Eng. and Ph.D. degrees in electronics and electrical engineering from the University of Glasgow, Glasgow, U.K. He is a Professor of Electrical Engineering with the King Abdullah University of Science and Technology, Thuwal, Saudi Arabia. His research focuses on the study of semiconductor lasers, LEDs, and photonic integrated circuits for applications in energy efficient lighting and visible-light communication. He is a Fellow of OSA, SPIE, and IOP (U.K.) and a Fellow of the U.S. National Academy of Inventors. He has served on the Editorial Board of IEEE PHOTONICS JOURNAL, and on the Technical Program Committee of IEDM, OFC, CLEO, and IPC. He is currently an Associate Editor for Optics Express and Journal of Nanophotonics. He is a Fellow of OSA, SPIE, and IOP (U.K.). He is also a Fellow of the U.S. National Academy of Inventors.

Michael Berumen (michael.berumen@kaust.edu.sa) obtained his B.S. (summa cum laude) in zoology at the University of Arkansas, U.S., and earned his doctoral degree in marine biology from James Cook University, Australia. Currently, he is a professor of marine science and the director of the KAUST Red Sea Research Center (RSRC). As a coral reef biologist, he is interested in all facets of ecology. Berumen's particular research interests focus on larval connectivity and dispersal of reef fishes, movement ecology of reef organisms, and the biodiversity and evolutionary biology of Red Sea fauna.

Mohamed Slim Alouini [F'09] (slim.alouini@kaust.edu.sa) received his $\mathrm{Ph} . \mathrm{D}$. degree in electrical engineering from the California Institute of Technology (Caltech), Pasadena, in 1998. He served as a faculty member at the University of Minnesota, Minneapolis, and then at Texas A\&M University at Qatar, Education City, Doha, before joining King Abdullah University of Science and Technology Thuwal, Makkah Province, Saudi Arabia, as a professor of electrical engineering in 2009. His current research interests include the modeling, design, and performance analysis of wireless communication systems. 